\title{
HARDY INEQUALITIES WITH BOUNDARY SINGULARITIES
}

\author{
CRISTIAN CAZACU
}

BCAM-Basque Center for Applied Mathematics, Biskaia Technology Park, Building 500, E-48160, Derio-Basque Country-Spain

and

Departamento de Matemáticas, Facultad de Ciencias, Universidad Autónoma de Madrid, 28049 Madrid, Spain

\begin{abstract}
In this work we prove some Hardy-Poincaré inequalities with quadratic singular potentials localized on the boundary of a smooth domain. Then, we consider conical domains with vertex on the singularity and we show upper and lower bounds for the corresponding optimal constants in the Hardy inequality. In particular, we prove the asymptotic behavior of the optimal constant when the slot of the cone tends to zero.
\end{abstract}

\section{INTRODUCTION}

Hardy inequalities represent a classical subject in which there has been intensive research in the recent past, mainly motivated by its applications to Partial Differential Equations (PDE's) and, more precisely, with the positivity of the Schrödinger operator

$$
L_{\lambda}:=-\Delta-\frac{\lambda}{|x|^{2}}, \text { with } \lambda \in \mathbb{R},
$$

involving the inverse square singular potential $1 /|x|^{2}$.

Inverse square potentials are interesting because of their criticality since they are homogeneous of degree -2 . They often appear in the linearization of critical nonlinear PDE's playing a crucial role in the asymptotic behaviour of branches of solutions in bifurcation problems (e.g. 10, 31, 33]). The operator $L_{\lambda}$ arises in physics and in particular in the relativity theory and quantum mechanics [16]. We also mention other interesting applications in molecular physics [27, quantum cosmology [4], combustion models [22], brownian motion [26], etc.

In [25], G. H Hardy proved that, in the one dimensional case, the optimal inequality

$$
\int_{0}^{\infty}\left|u^{\prime}(r)\right|^{2} d r \geq \frac{1}{4} \int_{0}^{\infty} \frac{u^{2}(r)}{r^{2}} d r
$$

E-mail address: cazacu@bcamath.org

Partially supported by the Grant MTM2008-03541 of the MICINN, Spain, the ERC Advanced Grant FP7246775 NUMERIWAVES, the Grant PI2010-04 of the Basque Government, and the doctoral fellowship FPUUAM from Universidad Autónoma de Madrid. 
holds for functions belonging to $H_{0}^{1}(0, \infty)$. More precisely,

$$
\inf _{u \in H_{0}^{1}(0, \infty)}\left(\int_{0}^{\infty}\left|u^{\prime}(r)\right|^{2} d r / \int_{0}^{\infty} \frac{u^{2}}{r^{2}} d r\right)=\frac{1}{4} .
$$

The classical multi-dimensional Hardy inequality (cf. [24]) asserts that for any $\Omega$ an open subset of $\mathbb{R}^{N}, N \geq 3$, it holds that

$$
\int_{\Omega}|\nabla u|^{2} d x \geq \frac{(N-2)^{2}}{4} \int_{\Omega} \frac{u^{2}}{|x|^{2}} d x
$$

for all $u \in H_{0}^{1}(\Omega)$. Moreover, if $\Omega$ contains the origin, the constant $(N-2)^{2} / 4$ is optimal and it is not attained. For $N=2$, inequality (1.4) is trivially true.

The reader interested in the existing literature on the extensions of the classical Hardy inequality is referred, in particular, to the following papers and the references therein: [22], [2], [1], [3], [18], [19], 32]. Recently, improved versions of (1.4) have been established in open bounded domains containing the origin (see [10], [33, [1]). We also mention the papers [6], 17] and the references therein for discussing inequalities with multipolar singularities. There has been also an intensive research for singular potentials involving the distance to the boundary (e.g. [8], [9]).

However, Hardy inequalities with one singular potential, in which the singularity lies on the boundary have been less investigated. This paper is mainly devoted to analyze this issue. To be more precise, throughout the paper, we consider $\Omega$ to be a subset of $\mathbb{R}^{N}$ with the origin $x=0$ placed on its boundary $\partial \Omega$, where the singularity is located. We then define $\mu(\Omega)$ as the best constant in the inequality

$$
\int_{\Omega}|\nabla u|^{2} d x \geq \mu(\Omega) \int_{\Omega} \frac{u^{2}}{|x|^{2}} d x, \quad \forall \quad u \in H_{0}^{1}(\Omega)
$$

i.e.

$$
\mu(\Omega):=\inf \left\{\int_{\Omega}|\nabla u|^{2} d x / \int_{\Omega} u^{2} /|x|^{2} d x, u \in H_{0}^{1}(\Omega)\right\} .
$$

Of course, in view of (eq167), $\mu(\Omega) \geq(N-2)^{2} / 4$. The authors in [28] showed that the strict inequality $\mu(\Omega)>(N-2)^{2} / 4$ holds true when $\Omega$ is a bounded domain of class $C^{2}$. Actually, the value $\mu(\Omega)$ depends on the geometric properties of the boundary $\partial \Omega$ at the singularity. The first explicit case has been given for $\Omega=\mathbb{R}_{+}^{N}$, where $\mathbb{R}_{+}^{N}$ is the half-space of $\mathbb{R}^{N}$ in which the condition $x_{N}>0$ holds. More precisely, for any $N \geq 1$, Filippas, Tertikas and Tidblom proved in 20] the new Hardy inequality:

$$
\int_{\mathbb{R}_{+}^{N}}|\nabla u|^{2} d x \geq \frac{N^{2}}{4} \int_{\mathbb{R}_{+}^{N}} \frac{u^{2}}{|x|^{2}} d x \quad \forall \quad u \in H_{0}^{1}\left(\mathbb{R}_{+}^{N}\right) .
$$

Moreover, they proved the constant $N^{2} / 4$ to be optimal (cf. Corollary 2.4, pp. 12, 20]) i.e. $\mu\left(\mathbb{R}_{+}^{N}\right)=N^{2} / 4$.

As a consequence of this result, mainly by the invariance under dilatations, it holds that $\mu(\Omega)=N^{2} / 4$ for any domain $\Omega$ of class $C^{2}$ with the support in the half-space $\mathbb{R}_{+}^{N}$. Moreover, if $\Omega$ is a bounded domain contained in the half-space, the following improved Hardy-Poincaré 
holds for any $u \in H_{0}^{1}(\Omega)$ (cf. [28]):

$$
\int_{\Omega}|\nabla u|^{2} d x-\frac{N^{2}}{4} \int_{\Omega} \frac{u^{2}}{|x|^{2}} d x \geq \frac{\lambda(\mathbb{D})}{|\operatorname{diam}(\Omega)|^{2}} \int_{\Omega} u^{2} d x
$$

where $\lambda_{1}(\Omega)$ is the first eigenvalue of the Laplacian in the unit ball in 2-d.

Another interesting situation appears in non-smooth domains $\Omega$, when the boundary develops corners or cusps at the singularity. The most relevant example of such a domain is represented by a cone with the vertex at the origin $x=0$. The question of studying the exact value of $\mu(\Omega)$ in cones has been full-filled in 2-d case. More precisely, if $\mathcal{C}_{\gamma}$ is the conical sector with the slot $\gamma \in(0,2 \pi)$, then (cf. [13])

$$
\mu\left(\mathcal{C}_{\gamma}\right)=\frac{\pi^{2}}{\gamma^{2}}
$$

By our knowledge, for higher dimensions $N \geq 3$, the value $\mu(\Omega)$ is only known when the cone $\Omega$ coincides with the half-space $\mathbb{R}_{+}^{N}$.

In this paper, the aim we focus on is two folded.

Roughly speaking, in the first part, we improve some results in [28] addressing HardyPoincaré inequalities in smooth domains. Besides, we complete rigorous proofs of several results stated in [28]. In the second part, the new issue addressed is related to optimal Hardy inequalities in conical domains in dimensions $N \geq 3$.

Part of the results in this paper were first announced in [15]. Soon after that, the preprint 28] has been submitted for publication while this extended version of the paper was being prepared. Because [28] yields some similar results, we thus present here in a detailed manner the most novel aspects of the note [15] not addressed in [28].

Let us now resume the content of the paper and the main results we obtain. In Section 2 we show Hardy-Poincaré inequalities in bounded smooth domains completing and extending some results in [28]. We mainly refer to Theorems 2.1, 2.2, 2.3. These results turn out to be closely related to the ellipticity of $\Omega$ at the origin, but also to the global geometry of $\Omega$. When $\Omega$ is not elliptic at the origin, a weaker Hardy inequality holds (cf. Theorem 2.4) and the continuous dependence of the Hardy constant in cones is required in the proof. This last result is rigorously shown in Section 3 , Theorem 3.1. Besides, in Section 3 we prove lower and upper bounds for the optimal constant $\mu(\Omega)$, when $\Omega$ is a cone in dimensions $N \geq 3$, with the vertex in $x=0$. In particular, the asymptotic value of $\mu(\Omega)$ is shown when the slot of the cone tends to zero (see Remark 3.1). We point out that all the sections contain at least a small introduction at the beginning. In Section 4 we conclude with some comments.

\section{INEQUALITIES IN SMOOTH DOMAINS}

As we said above, the value of the optimal constant $\mu(\Omega)$ depends on the various geometric properties of $\Omega$. In this section we assume $\Omega$ to be a Lipschitz domain with smooth boundary around the origin. Then $\partial \Omega$ is an $(N-1)$-Riemannian submanifold of $\mathbb{R}^{N}$ and assume that $\alpha_{1}, \alpha_{2}, \ldots, \alpha_{N-1}$ are the principal curvatures of $\partial \Omega$ at 0 . Then, up to a rotation (cf. [5], [23]), the boundary near the origin can be written as

$$
x_{N}=h\left(x^{\prime}\right)=\sum_{i=1}^{N-1} \alpha_{i} x_{i}^{2}+o\left(\left|x^{\prime}\right|^{2}\right) \text { as }\left|x^{\prime}\right| \rightarrow 0,
$$


where $x^{\prime}=\left(x_{1}, \ldots, x_{N-1}, 0\right)$. It is well-known that the principal curvatures are the eigenvalues of the $2 n d$ fundamental form of the surface $\partial \Omega$ (cf. [21]). If we choose

$$
\gamma<\min \left\{\alpha_{i}: 1 \leq i \leq N\right\}
$$

then $x_{N}>\gamma\left|x^{\prime}\right|^{2}$ in $\Omega$ for any $\left(x_{N}, x^{\prime}\right) \in \mathbb{R}^{N}$ very close to origin. Such points belong to the paraboloid $P_{\gamma}$ defined by

$$
P_{\gamma}=\left\{x=\left.\left(x^{\prime}, x_{N}\right) \in \mathbb{R}^{N}\left|x_{N}>\gamma\right| x^{\prime}\right|^{2}\right\} .
$$

Due to the considerations above, we distinguish the following four main situations.

C1. The elliptic case: There exists $\gamma>0$ such that $\Omega \subset P_{\gamma}$. In other words, $\Omega$ lies in the corresponding elliptic paraboloid $P_{\gamma}$ (see Figure 1, top left).

C2. The cylindrical case: $\Omega \subset P_{0}$, where $P_{0}=\mathbb{R}_{+}^{N}$. (see Figure 1, top right).

Remark 2.1. In cases $\mathrm{C} 1$ and $\mathrm{C} 2, \Omega$ lies in $\mathbb{R}_{+}^{N}$ (see Figure 1, top). From this point of view they may be analyzed together and the results that are true in $\mathrm{C} 2$ are also valid in $\mathrm{C} 1$. However, we analyze them separately because we present two independent tools to treat each of them.

C3. The locally elliptic case: In this case, $\Omega$ does not lie in $\mathbb{R}_{+}^{N}$, but this happens near the origin. More precisely, we suppose the existence of $\gamma_{\text {local }} \geq 0$ such that $\Omega \subset P_{\gamma_{\text {local }}}$ near the origin. Away from the origin we suppose that there exists $\gamma<0$ such that $\Omega \subset P_{\gamma}$ (see Figure 1, bottom left).

C4. The hyperbolic case: This corresponds to the situation when $\Omega$ has a hyperbolic geometry near the origin $x=0$. Therefore, we suppose that $\Omega \subset P_{\gamma}$ for some negative $\gamma<0$. (see Figure 1, bottom right).

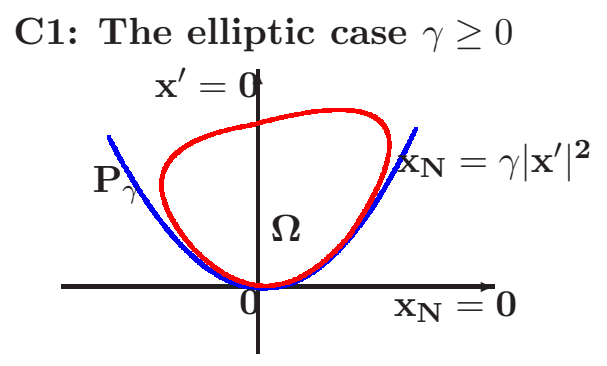

C3: The locally elliptic case $\gamma_{\text {loc }} \geq 0$



C2: The cylindrical case $\gamma=0$



C4: The hyperbolic case $\gamma<0$

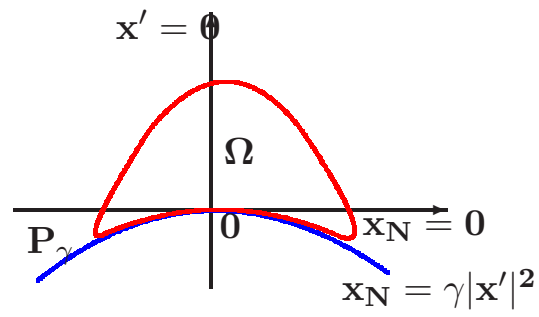

Figure 1. 
In the sequel we need the following technical lemma whose proof is given at the end of the section.

Lemma 2.1. Let $\Omega \subset P_{\gamma}$ be a domain fulfilling one of the conditions $\mathrm{C} 1-\mathrm{C} 4$ in Figure 1 , for some constant $\gamma \in \mathbb{R}$. Given $N \geq 2$ and $v \in H_{0}^{1}(\Omega)$, for any constant $C \in \mathbb{R}$, the function $u$ defined by

$$
u(x)=\frac{v(x)}{\left(x_{N}-\gamma\left|x^{\prime}\right|^{2}\right)}|x|^{C} .
$$

fulfills the following identity:

$$
\begin{aligned}
\int_{\Omega}|\nabla v|^{2} d x & =\int_{\Omega}\left(x_{N}-\gamma\left|x^{\prime}\right|^{2}\right)^{2}|x|^{-2 C}|\nabla u|^{2} d x+\left(C N-C^{2}\right) \int_{\Omega} \frac{v^{2}}{|x|^{2}} d x \\
& +2 \gamma \int_{\Omega}\left((N-1)|x|^{2}-C\left|x^{\prime}\right|^{2}\right)\left(x_{N}-\gamma\left|x^{\prime}\right|^{2}\right)|x|^{-2 C-2} u^{2} d x .
\end{aligned}
$$

2.1. Proof of main results. Next, we consider $\Omega$ to be a domain which satisfies the case C11 in Figure 1 (top, left). The main result in this case consists in an improvement of the Hardy-Poincaré inequality (1.7) shown in [28]. The proof is based on Lemma 2.1. More precisely, we have

Theorem 2.1. Let $N \geq 3$. Assume that $\Omega$ satisfies the condition $\mathrm{C} 1$. Then, for all $v \in H_{0}^{1}(\Omega)$ there exists a positive constant $C(\Omega, \gamma)$ such that

$$
\int_{\Omega}|\nabla v|^{2} d x-\frac{N^{2}}{4} \int_{\Omega} \frac{v^{2}}{|x|^{2}} d x \geq C(\Omega, \gamma) \int_{\Omega} \frac{v^{2}}{|x|} d x
$$

When $N=2$, the following weaker inequality holds

$$
\int_{\Omega}|\nabla v|^{2} d x \geq \frac{N^{2}}{4} \int_{\Omega} \frac{v^{2}}{|x|^{2}} d x
$$

Proof of Theorem 2.1. We put $C=N / 2$ in the identity (2.5) of Lemma 2.1. Taking into account that

$$
\max _{C \in \mathbb{R}}\left\{C N-C^{2}\right\}=\left.\left\{C N-C^{2}\right\}\right|_{C=N / 2}=\frac{N^{2}}{4},
$$

we obtain

$$
\int_{\Omega}|\nabla v|^{2} d x \geq \frac{N^{2}}{4} \int_{\Omega} \frac{v^{2}}{|x|^{2}} d x+2 \gamma \int_{\Omega}\left((N-1)|x|^{2}-\frac{N}{2}\left|x^{\prime}\right|^{2}\right)\left(x_{N}-\gamma\left|x^{\prime}\right|^{2}\right)|x|^{-N-2} u^{2} d x
$$

Using that

$$
(N-1)|x|^{2}-\frac{N}{2}\left|x^{\prime}\right|^{2} \geq \frac{N-2}{2}|x|^{2}
$$

we get

$$
\int_{\Omega}|\nabla v|^{2} d x \geq \frac{N^{2}}{4} \int_{\Omega} \frac{v^{2}}{|x|^{2}} d x+\gamma(N-2) \int_{\Omega} \frac{v^{2}(x)}{x_{N}-\gamma\left|x^{\prime}\right|^{2}} d x .
$$

We split the last term in two parts as follows:

$$
\int_{\Omega} \frac{v^{2}}{x_{N}-\gamma\left|x^{\prime}\right|^{2}} d x=\int_{\left\{x \in \Omega,\left|x^{\prime}\right| \leq 1 / \gamma\right\}} \frac{v^{2}}{x_{N}-\gamma\left|x^{\prime}\right|^{2}} d x+\int_{\left\{x \in \Omega,\left|x^{\prime}\right| \geq 1 / \gamma\right\}} \frac{v^{2}}{x_{N}-\gamma\left|x^{\prime}\right|^{2}} d x:=I_{1}+I_{2}
$$


In the first term, using that $\left|x^{\prime}\right| \leq 1 / \gamma$ implies $x_{N}-\gamma\left|x^{\prime}\right|^{2} \leq 2|x|$, we obtain

$$
I_{1} \geq \frac{1}{2} \int_{\left\{\left|x^{\prime}\right| \leq 1 / \gamma\right\}} \frac{v^{2}}{|x|} d x .
$$

Using the notation $R_{\Omega}=\sup _{x \in \bar{\Omega}}|x|$ we have $x_{N}-\gamma\left|x^{\prime}\right|^{2} \leq R_{\Omega}+\gamma R_{\Omega}^{2}$. Thus, for the second term we obtain

$$
I_{2} \geq \frac{1}{R_{\Omega}+\gamma R_{\Omega}^{2}} \int_{\left\{\left|x^{\prime}\right| \geq 1 / \gamma\right\}} v^{2} d x \geq \frac{1}{\gamma\left(R_{\Omega}+\gamma R_{\Omega}^{2}\right)} \int_{\left\{\left|x^{\prime}\right| \geq 1 / \gamma\right\}} \frac{v^{2}}{|x|} d x
$$

Combining these two lower bounds we get

$$
I_{1}+I_{2} \geq \min \left\{\frac{1}{2}, \frac{1}{\gamma\left(R_{\Omega}+\gamma R_{\Omega}^{2}\right)}\right\} \int_{\Omega} \frac{v^{2}}{|x|} d x
$$

and this, together with (2.9) yields (2.6). For $N=2$, (2.7) holds easily from (2.9).

Lemma 2.1 does not provide sufficient information for $\gamma=0$. However, using spherical harmonics decomposition, we can extend and improve the result of Theorem 2.1 to the case $\gamma \geq 0$ as follows.

Theorem 2.2. Let $N \geq 2$, and $\Omega \subset \mathbb{R}^{N}$ be such that the condition $\mathrm{C} 2$ is satisfied in Figure 1 (top, right). If $L$ is a positive number such that $L>\sup _{x \in \bar{\Omega}}|x|$, then for any $v \in H_{0}^{1}(\Omega)$,

$$
\int_{\Omega}|\nabla v|^{2} d x \geq \frac{N^{2}}{4} \int_{\Omega} \frac{v^{2}}{|x|^{2}} d x+\frac{1}{4} \int_{\Omega} \frac{v^{2}}{|x|^{2} \log ^{2}(L /|x|)} d x
$$

The following lemma will be necessary in the proof of Theorem 2.2 ,

Lemma 2.2. Let $L>R>0$. Then

$$
\int_{0}^{R}\left(w^{\prime}(r)\right)^{2} r d r \geq \frac{1}{4} \int_{0}^{R} \frac{w^{2}}{r^{2} \log ^{2}(L / r)} r d r, \quad \forall \quad w \in H_{0}^{1}(0, R) .
$$

The proof of Lemma 2.2 is given at the end of the section.

Proof of Theorem 2.2. Firstly, let us set $R=R_{\Omega}:=\sup _{x \in \bar{\Omega}}|x|$. such that $\Omega \subset B_{R}^{+}$where $B_{R}^{+}$ is the half ball of radius $R$

$$
B_{R}^{+}:=\left\{x \in \mathbb{R}^{N},|x| \leq R, x_{N} \geq 0\right\} .
$$

We consider also the lower half ball of radius $R$,

$$
B_{R}^{-}:=\left\{x \in \mathbb{R}^{N},|x| \leq R, x_{N} \leq 0\right\} .
$$

The proof follows the idea of decomposition in spherical harmonics (see [33]). By a density argument we can consider $v \in C_{0}^{1}\left(B_{R}^{+}\right)$. Building the odd extension

$$
u(x)=u\left(x_{1}, x_{2}, \ldots, x_{N}\right):= \begin{cases}v\left(x_{1}, x_{2}, \ldots, x_{N}\right), & x \in B_{R}^{+}, \\ -v\left(x_{1}, x_{2}, \ldots,-x_{N}\right), & x \in B_{R}^{-},\end{cases}
$$

we obtain $u \in C_{0}^{1}\left(B_{R}\right)$ and moreover,

$$
\int_{B_{R}^{+}}|\nabla v|^{2} d x=\frac{1}{2} \int_{B_{R}}|\nabla u|^{2} d x
$$




$$
\int_{B_{R}^{+}} \frac{v^{2}}{|x|^{2}} d x=\frac{1}{2} \int_{B_{R}} \frac{u^{2}}{|x|^{2}} d x
$$

Next we note that

$$
\int_{S^{N-1}} u(r, \sigma) d \sigma=0, \quad \forall \quad r \in[0,1] .
$$

Consider the expansion of $u$ in spherical harmonics

$$
u(x)=u(r, \sigma)=\sum_{k=0}^{\infty} u_{k}(r) f_{k}(\sigma) .
$$

Here $\left(f_{k}\right)_{k \geq 0}$ is an ortonormal basis of $L^{2}\left(S^{N-1}\right)$ constituted by the eigenvectors of the spherical Laplacian $\Delta_{S^{N-1}}$ with the corresponding eigenvalues $c_{k}=k(N+k-2), k \geq 0$. Here $S^{N-1}$ is the unit sphere with $(N-1)$-dimensional Hausdorff measure $N \omega_{N}$, where $\omega_{N}$ is the Lebesgue measure of the unit ball. It is well-known that $f_{0}$ is a constant. Integrating (2.15) on $S^{N-1}$ we get

$$
u_{0}(r)=\int_{S^{N-1}} u(r, \sigma) f_{0}(\sigma) d \sigma=f_{0}(\sigma) \int_{S^{N-1}} u(r, \sigma) d \sigma=0
$$

Therefore

$$
u(x)=u(r, \sigma)=\sum_{k=1}^{\infty} u_{k}(r) f_{k}(\sigma) .
$$

and by Plancherel identity we have

$$
\int_{B_{R}} u^{2} d x=N \omega_{N} \sum_{k=1}^{\infty} \int_{0}^{R}\left|u_{k}(r)\right|^{2} r^{N-1} d r .
$$

Using the representation of the Laplace operator in spherical coordinates

$$
\Delta=-\partial_{r}^{2}-\frac{N-1}{r} \partial_{r} u-\frac{1}{r^{2}} \Delta_{S^{N-1}}
$$

we get

$$
\int_{B_{R}}|\nabla u|^{2} d x=N \omega_{N} \sum_{k=1}^{\infty} \int_{0}^{R}\left[\left|u_{k}^{\prime}\right|^{2}+c_{k} \frac{u_{k}^{2}(r)}{r^{2}}\right] r^{N-1} d r .
$$

Let us denote $w_{k}(r)=u_{k}(r) r^{\frac{N-2}{2}}$ and $C_{N}:=(N-2)^{2} / 4$. Then by (2.18) we have

$$
\begin{aligned}
\int_{B_{R}}|\nabla u|^{2} d x & -\frac{N^{2}}{4} \int_{B_{R}} \frac{u^{2}}{|x|^{2}} d x=N \omega_{N} \sum_{k=1}^{\infty} \int_{0}^{R}\left[\left|u_{k}^{\prime}\right|^{2}-C_{N} \frac{u_{k}^{2}}{r^{2}}\right] r^{N-1} d r+ \\
& +N \omega_{N} \sum_{k=1}^{\infty}\left(c_{k}-(N-1)\right) \int_{0}^{R} \frac{u_{k}^{2}}{r^{2}} r^{N-1} d r \\
& \geq N \omega_{N} \sum_{k=1}^{\infty} \int_{0}^{R}\left[\left|u_{k}^{\prime}\right|^{2}-C_{N} \frac{u_{k}^{2}}{r^{2}}\right] r^{N-1} d r \\
& =N \omega_{N} \sum_{k=1}^{\infty} \int_{0}^{R}\left|w_{k}^{\prime}(r)\right|^{2} r d r
\end{aligned}
$$


Hence, by (2.19) and Lemma 2.2 we have

$$
\begin{aligned}
\int_{B_{R}}|\nabla u|^{2} d x-\frac{N^{2}}{4} \int_{B_{R}} \frac{u^{2}}{|x|^{2}} d x & \geq \frac{N \omega_{N}}{4} \sum_{k=1}^{\infty} \int_{0}^{R} \frac{w_{k}^{2}(r)}{r^{2} \log ^{2}(L / r)} r d r \\
& =\frac{N \omega_{N}}{4} \sum_{k=1}^{\infty} \int_{0}^{R} \frac{u_{k}^{2}(r)}{r^{2} \log ^{2}(L / r)} r^{N-1} d r .
\end{aligned}
$$

On the other hand

$$
N \omega_{N} \sum_{k=1}^{\infty} \int_{0}^{R} \frac{u_{k}^{2}(r)}{\left(r^{2} \log ^{2}(L / r)\right.} r^{N-1} d r=\int_{B_{R}} \frac{u^{2}}{|x|^{2} \log ^{2}(L /|x|)} d x .
$$

By (2.20), (2.21) and undoing the variables, the proof is completed.

Theorem 2.3. Let $N \geq 2$ and $\Omega$ be a domain satisfying the case $\mathrm{C} 3$ as in Figure 1 (bottom, left). Then, for any $v \in H_{0}^{1}(\Omega)$, there exists a constant $C(\Omega)$ such that

$$
C(\Omega) \int_{\Omega} v^{2} d x+\int_{\Omega}|\nabla v|^{2} d x \geq \frac{N^{2}}{4} \int_{\Omega} \frac{v^{2}}{|x|^{2}} d x+\frac{1}{4} \int_{\Omega} \frac{v^{2}}{|x|^{2} \log ^{2}(L /|x|)} d x,
$$

where $L>\sup _{x \in \bar{\Omega}}|x|$. Moreover, (2.22) is optimal in the sense that the term corresponding to the $L^{2}$-norm on the left hand side term cannot be disregarded.

Sketch of the proof of Theorem 2.3; We apply a standard cut-off argument so that the function $v$ can be split as $v=v_{1}+v_{2}$ where $v_{1}$ lies near the singularity and $v_{2}$ is supported away from it. In the neighborhood of $x=0$, we can apply the improved inequality of Theorem 2.2 corresponding to $v_{1}$. Outside the origin there are no singularities so that the potential $1 /|x|^{2}$ that appears in the inequality, is bounded by a constant depending only on $\Omega$ and the profile of the cut-off function. This fact makes the quantity $\int v_{2}^{2} /|x|^{2} d x$ to be bounded from above, up to a constant, by $\int v_{2}^{2} d x$. There is also an intermediate zone that we have to deal with, and more precisely where the profile of the cut-off functions has the gradient different by zero. In that part, it suffices to show that the cross term $\int \nabla v_{1} \cdot \nabla v_{2}$ is bounded from below, up to a constant, by $\int v^{2} d x$. Gluing these, the proof of (2.22) ends. We skip all the computations of the proof but for more details of a cut-off technique see e.g. [33], pp. 111.

Then, the necessity of the term in $L^{2}$-norm on the left hand side of (2.22) is a consequence of Proposition 2.1,

In the sequel we consider a domain $\Omega \subset \mathbb{R}^{N}$ as in Figure 1 (bottom, right). The result we obtain is stated as follows.

Theorem 2.4. Let $N \geq 2$ and assume that $\Omega$ satifies the condition $\mathrm{C} 4$ as in Figure 1 (bottom, right). For any $\varepsilon>0, \varepsilon<<1$, there exists a constant $C(\Omega, \varepsilon)$ such that the following inequality holds:

$$
C(\Omega, \varepsilon) \int_{\Omega} v^{2} d x+\int_{\Omega}|\nabla v|^{2} d x \geq\left(\frac{N^{2}}{4}-\varepsilon\right) \int_{\Omega} \frac{v^{2}}{|x|^{2}} d x, \quad \forall \quad v \in H_{0}^{1}(\Omega) .
$$

Moreover, (2.23) is optimal in the sense that the term in $L^{2}$-norm of the left hand side term cannot be disregarded.

Remark 2.2. Theorem 2.4 is stated also in [28]. The authors in [28] omit to show the continuous dependence of the Hardy constant in cones which plays a crucial role in the proof 
of this theorem. To make the things clear, we give a rigorous proof of this last result in Section 3.

Sketch of the proof of Theorem 2.4. The proof is based on local approximations of $\Omega$ around the origin, by conical sectors. We consider sectors that approximate the hyperplane $\gamma=0$ from below. In this analysis we use Hardy inequalities in cones and the continuous dependence of the Hardy constant, more precisely, Corollary 3.1 in Section 3 below. This allows improving Hardy inequalities near the origin. Cut-off arguments allow to glue Hardy inequalities derived near the origin with terms in $L^{2}$-norm, provided that the potential $1 /|x|^{2}$ is bounded far from the singularity $x=0$. Basically, this proves (2.23). For further details of a cut-off technique we refer to [33], pp. 111.

The necessity of the term in $L^{2}$-norm on the left hand side of (2.23) is a consequence of Proposition 2.1 stated below.

Proposition 2.1. There exist smooth bounded open sets $\Omega \subset \mathbb{R}_{+}^{N}, N \geq 2$, satisfying either C?3 or $\mathrm{C} 4$ and such that

$$
\mu(\Omega)<\frac{N^{2}}{4}
$$

Proof of Proposition 2.1. From the characterization of the first eigenvalue one can show the strict anti-monotonicity

$$
\mathcal{D}_{1} \subset \subset \mathcal{D}_{2} \Rightarrow \lambda\left(\mathcal{D}_{1}\right)>\lambda\left(\mathcal{D}_{2}\right)
$$

Next we take a cone $\mathcal{C}$ strictly larger than $\mathbb{R}_{+}^{N}$. From (2.25) and (3.1) we obtain

$$
\mu(\mathcal{C})<\mu\left(\mathbb{R}_{+}^{N}\right)=\frac{N^{2}}{4}
$$

Therefore, there exists $u \in C_{0}^{\infty}(\mathcal{C})$ such that

$$
\frac{\int_{\mathcal{C}}|\nabla u|^{2} d x}{\int_{\mathcal{C}} u^{2} /|x|^{2} d x}<\frac{N^{2}}{4}
$$

Denote $K:=\overline{\operatorname{supp} u}$. Then $K \subset \subset \mathcal{C}$ and $\operatorname{dist}(K, \partial \mathcal{C})>0$. Hence, we can build an open set $\Omega$ satisfying either $\mathrm{C} 3$ or $\mathrm{C} 4$, such that $K \subset \Omega \subset \mathcal{C}$. Hence, $u \in C_{0}^{\infty}(\Omega)$ and we get that

$$
\mu(\Omega) \leq \frac{\int_{\mathcal{C}}|\nabla u|^{2} d x}{\int_{\mathcal{C}}|u|^{2} /|x|^{2} d x}<\frac{N^{2}}{4} .
$$

The proof is completed.

\subsection{Proofs of useful Lemmas.}

Lemma 2.3. Let $N \geq 2$ and $\Omega \subset \mathbb{R}^{N}$ satisfying one of the conditions $\mathrm{C} 1-\mathrm{C} 4$. If $v \in H_{0}^{1}(\Omega)$ then

$$
\lim _{x \rightarrow \partial P_{\gamma}} \frac{v^{2}(x)}{x_{N}-\gamma\left|x^{\prime}\right|^{2}}=0 .
$$

Proof of Lemma 2.3. Extending $v$ with 0 outside $\Omega$ we get $v\left(x^{\prime}, \gamma\left|x^{\prime}\right|^{2}\right)=0$. Then

$$
0<\frac{v^{2}(x)}{x_{N}-\gamma\left|x^{\prime}\right|^{2}} \leq \int_{\gamma\left|x^{\prime}\right|^{2}}^{x_{N}}\left|\frac{\partial v}{\partial y_{N}}\left(x^{\prime}, y_{N}\right)\right|^{2} d y n
$$


which converges to 0 when $x_{N} \rightarrow \gamma\left|x^{\prime}\right|^{2}$. This is due to the properties of Lebesque integral and the fact that $\frac{\partial v}{\partial y_{N}}\left(x^{\prime}, \cdot\right)$ belongs to $L^{2}\left(\gamma\left|x^{\prime}\right|^{2}, x_{N}\right)$.

Proof of Lemma 2.1. Firstly

$$
\begin{gathered}
\nabla v=\left(x_{N}-\gamma\left|x^{\prime}\right|^{2}\right)|x|^{-C} \nabla u+u \nabla\left[\left(x_{N}-\gamma\left|x^{\prime}\right|^{2}\right)|x|^{-C}\right] \\
\nabla\left[\left(x_{N}-\gamma\left|x^{\prime}\right|^{2}\right)|x|^{-C}\right]=\sum_{i=1}^{N-1}\left[-2 \gamma x_{i}|x|^{-C}-C\left(x_{N}-\gamma\left|x^{\prime}\right|^{2}\right)|x|^{-C-2} x_{i}\right] e_{i}+ \\
+\left[|x|^{-C}-C x_{N}\left(x_{N}-\gamma\left|x^{\prime}\right|^{2}\right)|x|^{-C-2}\right] e_{N}
\end{gathered}
$$

where $\left\{e_{i}\right\}_{i=1, N}$ is canonical basis of $\mathbb{R}^{N}$. Then

$$
\begin{aligned}
\left|\nabla\left[\left(x_{N}-\gamma\left|x^{\prime}\right|^{2}\right)|x|^{-C}\right]\right|^{2} & =\left(1+4 \gamma^{2}\left|x^{\prime}\right|^{2}\right)|x|^{-2 C}+\left(C^{2}-2 C\right)\left(x_{N}-\gamma\left|x^{\prime}\right|^{2}\right)^{2}|x|^{-2 C-2}+ \\
& +2 \gamma C\left|x^{\prime}\right|^{2}\left(x_{N}-\gamma\left|x^{\prime}\right|^{2}\right)|x|^{-2 C-2}
\end{aligned}
$$

and

$$
\begin{aligned}
\operatorname{div}\left\{\left(x_{N}-\gamma\left|x^{\prime}\right|^{2}\right)|x|^{-C}\right. & \left.\nabla\left[\left(x_{N}-\gamma\left|x^{\prime}\right|^{2}\right)|x|^{-C}\right]\right\} \\
& =\left(2 C^{2}-C N-2 C\right)\left(x_{N}-\gamma\left|x^{\prime}\right|^{2}\right)^{2}|x|^{-2 C-2}+\left(1+4 \gamma^{2}\left|x^{\prime}\right|^{2}\right)|x|^{-2 C} \\
& -2\left[\gamma(N-1)|x|^{2}-2 \gamma C\left|x^{\prime}\right|^{2}\right]\left(x_{N}-\gamma\left|x^{\prime}\right|^{2}\right)|x|^{-2 C-2} .
\end{aligned}
$$

Using the formulas from above and integrating by parts we obtain

$$
\begin{aligned}
\int_{\Omega}|\nabla v|^{2} d x & =\int_{\Omega}|\nabla u|^{2}\left(x_{N}-\gamma\left|x^{\prime}\right|^{2}\right)^{2}|x|^{-2 C} d x+\int_{\Omega}\left|\nabla\left[\left(x_{N}-\gamma\left|x^{\prime}\right|^{2}\right)|x|^{-C}\right]\right|^{2} u^{2} d x+ \\
& +2 \int_{\Omega}\left(x_{N}-\gamma\left|x^{\prime}\right|^{2}\right)|x|^{-C} \nabla\left[\left(x_{N}-\gamma\left|x^{\prime}\right|^{2}\right)|x|^{-C}\right] u \nabla u d x \\
& =\int_{\Omega}|\nabla u|^{2}\left(x_{N}-\gamma\left|x^{\prime}\right|^{2}\right)^{2}|x|^{-2 C} d x+\int_{\Omega}\left|\nabla\left[\left(x_{N}-\gamma\left|x^{\prime}\right|^{2}\right)|x|^{-C}\right]\right|^{2} u^{2} d x \\
& +\int_{\partial \Omega} u^{2}\left(x_{N}-\gamma\left|x^{\prime}\right|^{2}\right)|x|^{-C} \nabla\left[\left(x_{N}-\gamma\left|x^{\prime}\right|^{2}\right)|x|^{-C}\right] \cdot \nu d \sigma- \\
& -\int_{\Omega} \operatorname{div}\left\{\left(x_{N}-\gamma\left|x^{\prime}\right|^{2}\right)|x|^{-C} \nabla\left[\left(x_{N}-\gamma\left|x^{\prime}\right|^{2}\right)|x|^{-C}\right]\right\} u^{2} d x
\end{aligned}
$$

Estimating the expression of the gradient in (2.28) we get

$$
\left|\nabla\left[\left(x_{N}-\gamma\left|x^{\prime}\right|^{2}\right)|x|^{-C}\right]\right|^{2} \leq C(\gamma, \Omega)|x|^{-2 C},
$$

where $C(\gamma, \Omega)$ is a suitable positive constant. Therefore, from (2.31) we have

$$
\left.\left|u^{2}\left(x_{N}-\gamma\left|x^{\prime}\right|^{2}\right)\right| x\right|^{-C} \nabla\left[\left(x_{N}-\gamma\left|x^{\prime}\right|^{2}\right)|x|^{-C}\right] \mid \leq C \frac{v^{2}(x)}{x_{N}-\gamma\left|x^{\prime}\right|^{2}},
$$

for some constant $C$. According to Remark 2.3 and (2.32) the boundary term of (2.30) vanishes and we obtain the new identity

$$
\begin{aligned}
\int_{\Omega}|\nabla v|^{2} d x & =\int_{\Omega}|\nabla u|^{2}\left(x_{N}-\gamma\left|x^{\prime}\right|^{2}\right)^{2}|x|^{-2 C}+\int_{\Omega}\left|\nabla\left[\left(x_{N}-\gamma\left|x^{\prime}\right|^{2}\right)|x|^{-C}\right]\right|^{2} u^{2} d x- \\
& -\int_{\Omega} \operatorname{div}\left\{\left(x_{N}-\gamma\left|x^{\prime}\right|^{2}\right)|x|^{-C} \nabla\left[\left(x_{N}-\gamma\left|x^{\prime}\right|^{2}\right)|x|^{-C}\right]\right\} u^{2} d x .
\end{aligned}
$$


By (2.28), (2.29) and (2.33) we have the identity (2.5). With this the proof of Lemma 2.1 ends.

Proof of Lemma 2.2. With the change of variables $w(r)=v(r) \log ^{1 / 2}(L / r)$ we have

$$
\left|w^{\prime}(r)\right|^{2}=\frac{1}{4 r^{2}} \log ^{-1}(L / r) v^{2}+v_{r}^{2} \log (L / r)-\frac{1}{r} v v_{r} .
$$

Therefore, due to the zero boundary conditions, we obtain

$$
\begin{aligned}
\int_{0}^{R}\left|w^{\prime}(r)\right|^{2} r d r & =\frac{1}{4} \int_{0}^{R} \frac{w^{2}}{r^{2} \log ^{2}(L / r)} r d r+\int_{0}^{R} v_{r}^{2} \log (L / r) r d r-\int_{0}^{R} v v_{r} d r \\
& =\frac{1}{4} \int_{0}^{R} \frac{w^{2}}{r^{2} \log ^{2}(L / r)} r d r+\int_{0}^{R} v_{r}^{2} \log (L / r) r d r \\
& \geq \frac{1}{4} \int_{0}^{R} \frac{w^{2}}{r^{2} \log ^{2}(L / r)} r d r .
\end{aligned}
$$

and Lemma 2.2 holds true.

\section{INEQUALITIES IN CONES}

Firstly, let us consider a Lipschitz connected cone $\mathcal{C} \subset \mathbb{R}^{N} \backslash\{0\}$ with the vertex at zero. Let $D \subset S^{N-1}$ be the Lipschitz domain such that

$$
\mathcal{C}=\{(r, \omega) \mid r \in(0, \infty), \omega \in \mathcal{D}\}
$$

Let $\mu(\mathcal{C})$ be the best constant in the Hardy inequality. Then (cf. [29])

$$
\mu(\mathcal{C})=\frac{(N-2)^{2}}{4}+\lambda_{1}(\mathcal{D})
$$

where $\lambda_{1}(\mathcal{D})$ is the Dirichlet principal eigenvalue of the spherical Laplacian $-\Delta_{S^{N-1}}$ on $\mathcal{D}$. In 2-d it is well-known that (e.g. [12])

$$
\lambda_{1}(\gamma):=\lambda_{1}(0, \gamma)=\pi^{2} / \gamma^{2},
$$

where $\gamma$ is the slot of the conical sector $\mathcal{C}_{\gamma}=\{(r, \omega) \mid r \in(0, \infty), \omega \in(0, \gamma)\}$ (see Figure

2 below). In higher dimensions $N \geq 3$, by our knowledge, $\lambda_{1}(\mathcal{D})$ is well-known only in the

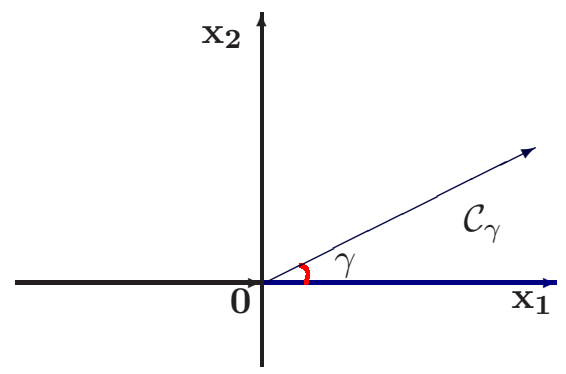

Figure 2. The 2-d conical sector with the aperture $\gamma$. 
case where $\mathcal{D}$ is the semi-sphere $S_{+}^{N-1}$ mapped in the upper half space $\mathbb{R}_{+}^{N}$. More precise, $\lambda_{1}\left(S_{+}^{N-1}\right)=N-1$. The half space $\mathbb{R}_{+}^{N}$ corresponds to the conical sector of slot $\gamma=\pi / 2$ (see Figure 3 below).

The aim of this section is mainly devoted to find lower bounds for $\lambda_{1}(\mathcal{D})$ in higher dimensions $N \geq 3$. In that sense, the definition of a cone in polar coordinates will be used.

3.1. The $N-d$ case, $N \geq 3$. For $0<\gamma<\pi$ we define the $N$-dimensional cone, with slot $\gamma$, denoted by $\mathcal{C}_{\gamma}$ (Figure 2), consisting in all $x=\left(x_{1}, x_{2}, \ldots, x_{N}\right) \in \mathbb{R}^{N}$ such that, in spherical coordinates (cf. [30, pp. 293),

$$
\mathcal{C}_{\gamma}:\left\{\begin{array}{l}
x_{1}=r \sin \theta_{1} \sin \theta_{2} \ldots \sin \theta_{N-2} \cos _{N-1} \\
x_{2}=r \sin \theta_{1} \sin \theta_{2} \ldots \sin \theta_{N-2} \sin _{N-1} \\
\vdots \\
x_{N-1}=r \sin \theta_{1} \sin \theta_{2} \\
x_{N}=r \cos \theta_{1}
\end{array}\right.
$$

with $r>0$ and

$$
\left\{\begin{array}{l}
0<\theta_{1} \leq \gamma \\
0 \leq \theta_{i} \leq \pi, \\
0 \leq \theta_{N-1} \leq 2 \pi
\end{array} \quad \text { for } 2 \leq i \leq N-2\right.
$$

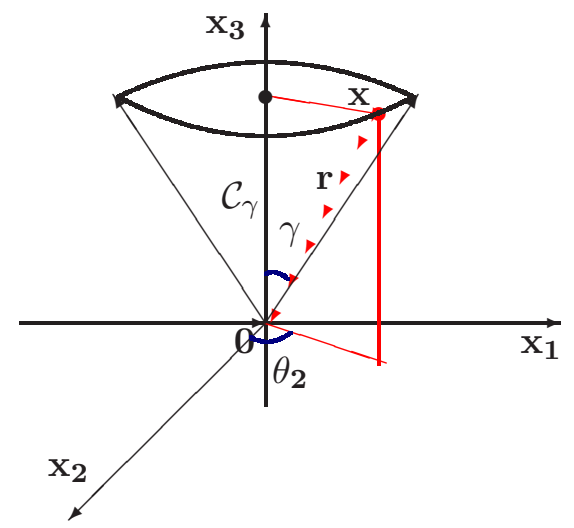

Figure 3. The cone with the slot $\gamma$

For simplicity we denote by $\lambda_{1}(\gamma):=\lambda_{1}\left(D_{\gamma}\right)$ the first Dirichlet eigenvalue of the spherical Laplacian on $\mathcal{D}_{\gamma}:=\mathcal{C}_{\gamma} \cap S^{N-1}$. Then we have

$$
\mu\left(\mathcal{C}_{\gamma}\right)=\frac{(N-2)^{2}}{4}+\lambda_{1}(\gamma)
$$

\subsection{Main results.}

Theorem 3.1. Assume that $N \geq 3$.

(a). If $0<\gamma \leq \frac{\pi}{2}$ then

$$
\lambda_{1}(\gamma) \geq \frac{(N-1) \pi^{2}}{4 \gamma^{2}}
$$


(b). For any $\varepsilon>0, \varepsilon<<1$, there exists $\delta=\delta(\varepsilon)>0$ such that for all $\frac{\pi}{2} \leq \gamma \leq \frac{\pi}{2}+\delta$

$$
\lambda_{1}(\gamma)>N-1-\varepsilon
$$

Corolary 3.1 (The continuous dependance of the Hardy constant).

$$
\lim _{\gamma \rightarrow \pi / 2} \mu\left(\mathcal{C}_{\gamma}\right)=\mu\left(\mathcal{C}_{\pi / 2}\right)=\frac{N^{2}}{4}
$$

Theorem 3.2. Assume $\gamma \in(0, \pi)$. Then it holds

$$
\left(\frac{\sin \gamma}{\gamma}\right)^{N-2}\left(\frac{B_{1}}{\gamma}\right)^{2} \leq \lambda_{1}(\gamma) \leq\left(\frac{\gamma}{\sin \gamma}\right)^{N-2}\left(\frac{B_{1}}{\gamma}\right)^{2}
$$

where $B_{1}$ is the first positive zero of the Bessel function $J_{\frac{N-3}{2}}$, of fractional order $(N-3) / 2$.

Remark 3.1 (Asymptotic behavior). From Theorem 3.2 above we get the asymptotic formula

$$
\lim _{\gamma \rightarrow 0} \frac{\lambda_{1}(\gamma) \gamma^{2}}{B_{1}^{2}}=1
$$

3.3. Preliminaries. Let us consider $u \in C_{c}^{\infty}\left(\mathcal{C}_{\gamma}\right)$. Then, in polar coordinates we have

$$
u\left(r, \theta_{1}, \theta_{2}, \ldots, \theta_{N-3}, \theta_{N-2}\right) \in C^{\infty}((0, \infty) \times(0, \gamma) \times(0, \pi) \times \ldots \times(0, \pi) \times(0,2 \pi)),
$$

vanishing in the neighborhoods of $r=\infty$ and $\theta_{1}=\gamma$.

The representation of the gradient in polar coordinates is given by

$$
\begin{aligned}
|\nabla u|^{2} & =\left|u_{r}\right|^{2}+\frac{u_{\theta_{1}}^{2}}{r^{2}}+\frac{u_{\theta_{2}}^{2}}{r^{2} \sin ^{2} \theta_{1}}+\frac{u_{\theta_{3}}^{2}}{r^{2} \sin ^{2} \theta_{1} \sin ^{2} \theta_{2}}+ \\
& +\ldots+\frac{u_{\theta_{N-1}}^{2}}{r^{2} \sin ^{2} \theta_{1} \sin ^{2} \theta_{2} \ldots \sin ^{2} \theta_{N-2}} .
\end{aligned}
$$

In other words,

$$
|\nabla u|^{2}=\left|u_{r}\right|^{2}+\frac{u_{\theta_{1}}^{2}}{r^{2}}+\text { positive terms. }
$$

The determinant of the Jacobian of the transformation has the form

$$
J\left(r, \theta_{1}, \theta_{2} \ldots, \theta_{N-2}\right)=r^{N-1} \sin ^{N-2} \theta_{1} \sin ^{N-3} \theta_{2} \ldots \sin \theta_{N-2} .
$$

To simplify the notations, we define the integral in the variables $\theta_{2}, \ldots, \theta_{N-2}$ as

$$
\int:=\int_{0}^{\pi} \ldots \int_{0}^{\pi} \int_{0}^{2 \pi} \sin ^{N-3} \theta_{2} \ldots \sin \theta_{N-2} \mathrm{~d} \theta_{2} \ldots \mathrm{d} \theta_{N-2} \mathrm{~d} \theta_{N-1} .
$$

For a fixed $\sigma \in S^{N-1} \cap \mathcal{C}_{\gamma}$ the radial function $r \mapsto u(\cdot, \sigma)$ satisfies the well-known Hardy inequality

and therefore

$$
\int_{0}^{\infty} u_{r}^{2} r^{N-1} d r \geq\left(\frac{N-2}{2}\right)^{2} \int_{0}^{\infty} \frac{u^{2}}{r^{2}} r^{N-1} d r
$$

$$
\int_{0}^{\infty} \int_{0}^{\gamma} \int u_{r}^{2} r^{N-1} \sin ^{N-2} \theta_{1} \mathrm{~d} \theta_{1} d r \geq\left(\frac{N-2}{2}\right)^{2} \int_{0}^{\infty} \int_{0}^{\gamma} \int \frac{u^{2}}{r^{2}} r^{N-1} \sin ^{N-2} \theta_{1} \mathrm{~d} \theta_{1} d r .
$$


From above, due to the lack of the boundary conditions in the variables $\theta_{2}, \ldots, \theta_{N-2}$ we get that $\lambda_{1}(\gamma)$ is the optimal constant in the weighted inequality

$$
\int_{0}^{\infty} \int_{0}^{\gamma} \int \frac{u_{\theta_{1}}^{2}}{r^{2}} r^{N-1} \sin ^{N-2} \theta_{1} \mathrm{~d} \theta_{1} d r \geq \lambda_{1}(\gamma) \int_{0}^{\infty} \int_{0}^{\gamma} \int \frac{u^{2}}{r^{2}} r^{N-1} \sin ^{N-2} \theta_{1} \mathrm{~d} \theta_{1} d r .
$$

More precisely, $\lambda_{1}(\gamma)$ may be characterized by

$$
\lambda_{1}(\gamma)=\inf _{\{u \in H, u \neq 0\}} \frac{\int_{0}^{\gamma} u_{\theta_{1}}^{2} \sin ^{N-2} \theta_{1} \mathrm{~d} \theta_{1}}{\int_{0}^{\gamma} u^{2} \sin ^{N-2} \theta_{1} \mathrm{~d} \theta_{1}},
$$

where $H$ is the completion of the space

$$
\left\{u \in C^{\infty}[0, \gamma) \mid u \text { vanishes in a neighborgood of } \gamma\right\}
$$

in the norm

$$
\|u\|_{H}^{2}=\int_{0}^{\gamma} u_{\theta_{1}}^{2} \sin ^{N-2} \theta_{1} \mathrm{~d} \theta_{1}
$$

Indeed, if $a_{n}=a_{n}(\theta)$ is an approximating sequence in (3.11) then the sequence

$$
u_{n}:=u_{1}(r) a_{n}\left(\theta_{1}\right) u_{2}\left(\theta_{2}\right) \ldots u_{N-2}\left(\theta_{N-2}\right),
$$

where $u_{1}$ is smooth and vanishes in the neighborhood of $r=\infty$, minimizes also $\lambda_{1}(\gamma)$.

\subsection{Proofs of Theorems.}

Proof of Theorem 3.1(泊). Without losing the generality, we are going to consider $u$ as in (3.7). Next we propose the change of variables

$$
v\left(r, \theta_{1}, \theta_{2}, \ldots, \theta_{N-2}\right):=u\left(r, \theta_{1}, \theta_{2}, \ldots, \theta_{N-2}\right) / \cos \left(\frac{\pi}{2 \gamma} \theta_{1}\right) .
$$

For simplicity, we write $u(r)$ or $u\left(\theta_{1}\right)$ when referring to the radial variable respectively at the angular part $\theta_{1}$. Thus, integrating by parts we get the following identity:

$$
\begin{aligned}
\int_{0}^{\gamma} u_{\theta_{1}}^{2}\left(\theta_{1}\right) \sin ^{N-2} \theta_{1} \mathrm{~d} \theta_{1} & =\int_{0}^{\gamma} v_{\theta_{1}}^{2}\left(\theta_{1}\right) \cos ^{2}\left(\frac{\pi}{2 \gamma} \theta_{1}\right) \sin ^{N-2} \theta_{1} \mathrm{~d} \theta_{1}+\frac{\pi^{2}}{4 \gamma^{2}} \int_{0}^{\gamma} u^{2}\left(\theta_{1}\right) \sin ^{N-2} \theta_{1} \mathrm{~d} \theta_{1} \\
& +(N-2) \frac{\pi}{2 \gamma} \int_{0}^{\gamma} v^{2}\left(\theta_{1}\right) \sin \left(\frac{\pi}{2 \gamma} \theta_{1}\right) \cos \left(\frac{\pi}{2 \gamma} \theta_{1}\right) \cos \theta_{1} \sin ^{N-3} \theta_{1} \mathrm{~d} \theta_{1} .
\end{aligned}
$$

Using the identity (3.13) and the characterization of $\lambda_{1}(\gamma)$ stated in (3.11), it is enough to show that

$$
\cos \left(\frac{\pi}{2 \gamma} \theta_{1}\right) \sin \left(\frac{\pi}{2 \gamma} \theta_{1}\right) \cos \theta_{1} \geq \frac{\pi}{2 \gamma} \cos ^{2}\left(\frac{\pi}{2 \gamma} \theta_{1}\right) \sin \theta_{1}, \quad \forall \quad \theta_{1} \in[0, \gamma] .
$$

Obviously, this is true for $\theta_{1}=\{0, \gamma\}$. Dividing by $\frac{\pi}{2 \gamma} \theta_{1} \cos ^{2}\left(\frac{\pi}{2 \gamma} \theta_{1}\right) \cos \theta_{1}$ it remains to prove that

$$
\frac{\tan \left(\frac{\pi}{2 \gamma} \theta_{1}\right)}{\frac{\pi}{2 \gamma} \theta_{1}} \geq \frac{\tan \theta_{1}}{\theta_{1}}, \quad \forall \quad \theta_{1} \in(0, \gamma) \text {. }
$$

Because $\pi / 2 \gamma>1$, the last inequality is true due to the increasing monotonicity of the function $\theta_{1} \mapsto \tan \theta_{1} / \theta_{1}$ in the interval $(0, \pi / 2)$. This ends the proof. 
Proof of Theorem [3.1([6). In the sequel we will use the notations

$$
\gamma=\pi / 2+\delta \quad \text { and } \bar{\gamma}:=\pi / 2+\delta / 2,
$$

where $\delta>0$. It suffices to prove that

Proposition 3.1. For any $\varepsilon>0, \varepsilon<<1$, there exists $\delta=\delta(\varepsilon)>0$, such that

$$
\int_{0}^{\bar{\gamma}(\varepsilon)} u_{\theta_{1}}^{2}\left(\theta_{1}\right) \sin ^{N-2} \theta_{1} \mathrm{~d} \theta_{1} \geq(N-1-\varepsilon) \int_{0}^{\bar{\gamma}(\varepsilon)} u^{2}\left(\theta_{1}\right) \sin ^{N-2} \theta_{1} \mathrm{~d} \theta_{1},
$$

for all $u \in H$.

Next we prove Proposition 3.1. Denote

$$
v\left(\theta_{1}\right):=u\left(\theta_{1}\right) / \cos \left(\frac{\pi}{2 \gamma} \theta_{1}\right)
$$

and similar to (3.13) we obtain

$$
\begin{aligned}
& \int_{0}^{\bar{\gamma}} u_{\theta_{1}}^{2}\left(\theta_{1}\right) \sin ^{N-2} \theta_{1} \mathrm{~d} \theta_{1}=\int_{0}^{\bar{\gamma}} v_{\theta_{1}}^{2}\left(\theta_{1}\right) \cos ^{2}\left(\frac{\pi}{2 \gamma} \theta_{1}\right) \sin ^{N-2} \theta_{1} \mathrm{~d} \theta_{1}+\frac{\pi^{2}}{4 \gamma^{2}} \int_{0}^{\bar{\gamma}} u^{2}\left(\theta_{1}\right) \sin ^{N-2} \theta_{1} \mathrm{~d} \theta_{1} \\
&+(N-2) \frac{\pi}{2 \gamma} \int_{0}^{\bar{\gamma}} v^{2}\left(\theta_{1}\right) \sin \left(\frac{\pi}{2 \gamma} \theta_{1}\right) \cos \left(\frac{\pi}{2 \gamma} \theta_{1}\right) \cos \theta_{1} \sin ^{N-3} \theta_{1} \mathrm{~d} \theta_{1} .
\end{aligned}
$$

Then

$$
\begin{aligned}
\int_{0}^{\bar{\gamma}} u_{\theta_{1}}^{2}\left(\theta_{1}\right) \sin ^{N-2} \theta_{1} \mathrm{~d} \theta_{1} & \geq \frac{\pi^{2}}{4 \gamma^{2}} \int_{0}^{\bar{\gamma}} u^{2}\left(\theta_{1}\right) \sin ^{N-2} \theta_{1} \mathrm{~d} \theta_{1} \\
& +(N-2) \frac{\pi}{2 \gamma} \int_{0}^{\bar{\gamma}} v^{2}\left(\theta_{1}\right) \sin \left(\frac{\pi}{2 \gamma} \theta_{1}\right) \cos \left(\frac{\pi}{2 \gamma} \theta_{1}\right) \cos \theta_{1} \sin ^{N-3} \theta_{1} \mathrm{~d} \theta_{1} \\
& :=A+B .
\end{aligned}
$$

Let $\varepsilon>0, \varepsilon<<1$ to be fixed. In order to compute $B$, we split it in two parts as

$$
B=(N-2) \frac{\pi}{2 \gamma} \int_{0}^{\pi / 2-\varepsilon} \ldots+(N-2) \frac{\pi}{2 \gamma} \int_{\pi / 2-\varepsilon}^{\bar{\gamma}} \ldots:=B_{1}+B_{2} .
$$

where Next we concentrate on $B_{1}$.

Firstly, there exists $\delta=\delta(\varepsilon)>0$, such that (cf. Lemma 3.2)

$$
\sin \left(\frac{\pi}{2 \gamma(\varepsilon)} \theta_{1}\right) \cos \theta_{1} \geq(1-\varepsilon) \cos \left(\frac{\pi}{2 \gamma(\varepsilon)} \theta_{1}\right) \sin \theta_{1}, \quad \forall \quad 0 \leq \theta_{1} \leq \frac{\pi}{2}-\varepsilon .
$$

Using this and undoing the variables we reach to

$$
\begin{aligned}
B_{1} & \geq(N-2) \frac{\pi}{2 \gamma(\varepsilon)}(1-\varepsilon) \int_{0}^{\pi / 2-\varepsilon} u^{2}\left(\theta_{1}\right) \sin ^{N-2} \theta_{1} \mathrm{~d} \theta_{1} \\
& =(N-2) \frac{\pi}{2 \gamma(\varepsilon)}(1-\varepsilon) \int_{0}^{\bar{\gamma}(\varepsilon)} u^{2}\left(\theta_{1}\right) \sin ^{N-2} \theta_{1} \mathrm{~d} \theta_{1}-B_{11},
\end{aligned}
$$

where

$$
B_{11}=(N-2) \frac{\pi}{2 \gamma(\varepsilon)}(1-\varepsilon) \int_{\pi / 2-\varepsilon}^{\bar{\gamma}(\varepsilon)} u^{2}\left(\theta_{1}\right) \sin ^{N-2} \theta_{1} \mathrm{~d} \theta_{1}
$$


and thus

$$
\left|B_{11}\right| \leq C_{1} \int_{\pi / 2-\varepsilon}^{\bar{\gamma}(\varepsilon)} u^{2}\left(\theta_{1}\right) \mathrm{d} \theta_{1}
$$

for some positive constant $C_{1}$ independent of $\varepsilon$. Undoing the variables we have

$$
B_{2}=(N-2) \frac{\pi}{2 \gamma(\varepsilon)} \int_{\pi / 2-\varepsilon}^{\bar{\gamma}(\varepsilon)} u^{2}\left(\theta_{1}\right)\left(\frac{\cos \theta_{1}}{\cos \left(\frac{\pi}{2 \gamma(\varepsilon)} \theta_{1}\right)}\right) \sin \left(\frac{\pi}{2 \gamma(\varepsilon)} \theta_{1}\right) \sin ^{N-3} \theta_{1} \mathrm{~d} \theta_{1} .
$$

Again, there exists $\delta=\delta(\varepsilon)>0$ and a constant $C$ independent of $\varepsilon$, such that (cf. Lemma 3.3)

$$
\left|\frac{\cos \theta_{1}}{\cos \left(\frac{\pi}{2 \gamma(\varepsilon)} \theta_{1}\right)}\right|<C, \quad \forall \quad 0 \leq \theta_{1} \leq \bar{\gamma}(\varepsilon),
$$

and reconsidering the constant $C$ we obtain

$$
\left|B_{2}\right| \leq C \int_{\pi / 2-\varepsilon}^{\bar{\gamma}(\varepsilon)} u^{2}\left(\theta_{1}\right) \mathrm{d} \theta_{1}
$$

From (3.19) and (3.20) we deduce

$$
\left|B_{11}\right|+\left|B_{2}\right| \leq C \int_{\pi / 2-\varepsilon}^{\bar{\gamma}(\varepsilon)} u^{2}\left(\theta_{1}\right) \mathrm{d} \theta_{1}
$$

On the other hand, from Leibnitz-Newton formula there exists $\tau=\tau(\varepsilon) \in(\pi / 2-\varepsilon, \bar{\gamma}(\varepsilon))$ such that

$$
\int_{\pi / 2-\varepsilon}^{\bar{\gamma}(\varepsilon)} u^{2}\left(\theta_{1}\right) \mathrm{d} \theta_{1}=(\bar{\gamma}(\varepsilon)+\varepsilon-\pi / 2) u^{2}(\tau(\varepsilon))=(\delta(\varepsilon) / 2+\varepsilon) u^{2}(\tau(\varepsilon)) .
$$

Applying Holder inequality we find

$$
u^{2}(\tau(\varepsilon))=\left|\int_{\tau(\varepsilon)}^{\bar{\gamma}(\varepsilon)} u_{\theta_{1}}\left(\theta_{1}\right) \mathrm{d} \theta_{1}\right|^{2} \leq C \int_{\tau(\varepsilon)}^{\bar{\gamma}(\varepsilon)} u_{\theta_{1}}^{2}\left(\theta_{1}\right) \mathrm{d} \theta_{1} \leq C_{1} \int_{0}^{\bar{\gamma}(\varepsilon)} u_{\theta_{1}}^{2}\left(\theta_{1}\right) \sin ^{N-2} \theta_{1} \mathrm{~d} \theta_{1},
$$

where $C, C_{1}$ are some positive constants independent of $\varepsilon$. From (3.21), (3.23) and (3.22) we deduce

$$
\left|B_{11}\right|+\left|B_{2}\right| \leq C(\delta(\varepsilon)+\varepsilon) \int_{0}^{\bar{\gamma}(\varepsilon)} u_{\theta_{1}}^{2}\left(\theta_{1}\right) \sin ^{N-2} \theta_{1} \mathrm{~d} \theta_{1} .
$$

Hence, according to (3.16) we get

$$
\begin{aligned}
(1-C(\delta(\varepsilon)+\varepsilon)) & \int_{0}^{\bar{\gamma}(\varepsilon)} u_{\theta_{1}}^{2}\left(\theta_{1}\right) \sin ^{N-2} \theta_{1} \mathrm{~d} \theta_{1} \geq \\
& \geq\left(\frac{\pi^{2}}{4 \gamma^{2}(\varepsilon)}+(N-2) \frac{\pi}{2 \gamma(\varepsilon)}(1-\varepsilon)\right) \int_{0}^{\bar{\gamma}(\varepsilon)} u^{2}\left(\theta_{1}\right) \sin ^{N-2} \theta_{1} \mathrm{~d} \theta_{1} .
\end{aligned}
$$

In other words

$$
\int_{0}^{\gamma} u_{\theta_{1}}^{2}\left(\theta_{1}\right) \sin ^{N-2} \theta_{1} \mathrm{~d} \theta_{1} \geq \frac{\frac{\pi^{2}}{4 \gamma^{2}(\varepsilon)}+(N-2) \frac{\pi}{2 \gamma(\varepsilon)}(1-\varepsilon)}{1-C(\delta(\varepsilon)+\varepsilon)} \int_{0}^{\gamma} u^{2}\left(\theta_{1}\right) \sin ^{N-2} \theta_{1} \mathrm{~d} \theta_{1} .
$$


Due to the fact that

$$
\lim _{\delta \searrow 0} \frac{\frac{\pi^{2}}{4 \gamma^{2}}+(N-2) \frac{\pi}{2 \gamma}(1-\varepsilon)}{1-C(\delta+\varepsilon)}=\frac{N-1-(N-2) \varepsilon}{1-C \varepsilon},
$$

we may reconsider $\delta=\delta(\varepsilon)>0$ from above such that

$$
\frac{\frac{\pi^{2}}{4 \gamma^{2}(\varepsilon)}+(N-2) \frac{\pi}{2 \gamma(\varepsilon)}(1-\varepsilon)}{1-C(\delta(\varepsilon)+\varepsilon)}>\frac{N-1-(N-2) \varepsilon}{1-C \varepsilon}-\frac{\varepsilon}{2} .
$$

Now we can choose $\varepsilon>0$ small enough such that

$$
\frac{N-1-(N-2) \varepsilon}{1-C \varepsilon} \geq N-1-\frac{\varepsilon}{2},
$$

and for each $\delta=\delta(\varepsilon)$ from above, we have

$$
\frac{\frac{\pi^{2}}{4 \gamma^{2}(\varepsilon)}+(N-2) \frac{\pi}{2 \gamma(\varepsilon)}(1-\varepsilon)}{1-C(\delta(\varepsilon)+\varepsilon)}=\frac{N-1}{1-C \varepsilon}>N-1-\varepsilon .
$$

We end up the proof of the second part of Theorem 3.1.

Proof of Theorem [3.2. with $\gamma \in(0, \pi)$. Next, the aim is to show upper and lower bounds for the value $\lambda_{1}(\gamma)$ in (3.11). Due to inequalities

$$
\frac{\sin \gamma}{\gamma} t \leq \sin t \leq t, \quad \forall \quad t \in(0, \gamma), \gamma \in(0, \pi),
$$

it suffices to determine the value of

$$
\lambda_{\star}^{1}(\gamma):=\inf _{\{u \in H, u \neq 0\}} \frac{\int_{0}^{\gamma} u_{t}^{2} t^{N-2} d t}{\int_{0}^{\gamma} u^{2} t^{N-2} d t},
$$

which is well defined in $H$ since

$$
\int_{0}^{\gamma} u_{t}^{2} t^{N-2} d t<\infty \text { iff } \int_{0}^{\gamma} u_{t} \sin ^{N-2} t d t<\infty
$$

$H$ being defined by the norm in (3.12). By Proposition 3.2, it holds that $\lambda_{\star}^{1}(\gamma)>0$. Due to the compact embedding (see Proposition 3.3 )

$$
H \hookrightarrow L^{2}\left([0, \gamma], t^{N-2} d t\right),
$$

$\lambda_{\star}^{1}(\gamma)$ is attained by a non-trivial function $\phi_{1}$. Then one can prove that $\lambda_{1}^{\star}(\gamma)$ satisfies the variational problem: there exists $\phi^{1} \in H$ such that

$$
\int_{0}^{\gamma} \phi_{t}^{1} v_{t} t^{N-2} d t=\lambda_{1}^{\star}(\gamma) \int_{0}^{\gamma} \phi^{1} v t^{N-2} d t, \quad \forall \quad v \in H .
$$

Next we note that any $u \in H$ exhibits a hidden weak Neumann boundary condition at the origin $t=0$ :

$$
\lim _{\varepsilon \rightarrow 0} \frac{1}{\varepsilon} \int_{0}^{\varepsilon} u_{t} t^{N-2} d t=0
$$

Indeed, we have

$$
\left|\frac{1}{\varepsilon} \int_{0}^{\varepsilon} u_{t} t^{N-2} d t\right| \leq \frac{1}{\varepsilon}\left(\int_{0}^{\varepsilon}\left(u_{t}\right)^{2} t^{N-2} d t\right)^{1 / 2}\left(\int_{0}^{\varepsilon} t^{N-2} d t\right)^{1 / 2}=\varepsilon^{(N-3) / 2}\left(\int_{0}^{\varepsilon} u_{t} t^{N-2} d t\right)^{1 / 2}
$$


which converges to 0 when $\varepsilon$ tends to 0 . This allows to make integrations by parts and rewrite (3.29) as

$$
\int_{0}^{\gamma}-\left(\phi_{t}^{1} t^{N-2}\right)_{t} v d t=\lambda_{1}^{\star}(\gamma) \int_{0}^{\gamma} \phi^{1} v t^{N-2} d t, \quad \forall \quad v \in H .
$$

Therefore, $\lambda_{\star}^{1}(\gamma)$ is the first eigenvalue of the degenerate Sturm-Liouville problem

$$
\left\{\begin{array}{l}
-\left(u_{t} t^{N-2}\right)_{t}=\lambda u t^{N-2}, \quad t \in(0, \gamma), \\
\lim _{t \rightarrow 0} u_{t} t^{N-2}=0, \quad u(\gamma)=0,
\end{array}\right.
$$

with the corresponding eigenvector $\phi_{1}$.

In the sequel we determine explicitly the value of $\lambda_{\star}^{1}(\gamma)$.

With the change of variables $v=u t^{N-2}$, the problem (3.33) reduces to the following Bessel equation with boundary constraint

$$
\left\{\begin{array}{l}
v_{t t}+(2-N) \frac{v_{t}}{t}+\left(\lambda+\frac{N-2}{t^{2}}\right) v=0, \quad t \in(0, \gamma), \\
v(\gamma)=0
\end{array}\right.
$$

3.4.1. Bessel functions. If $n$ is positive integer then, the first Bessel function $J_{n}$ of order $n$ has the expression

$$
J_{n}(x)=\frac{x^{n}}{2^{n} n !}\left(1-\frac{x^{2}}{2 \cdot(2 n+2)}+\frac{x^{4}}{2 \cdot 4 \cdot(2 n+2) \cdot(2 n+4)}-\ldots\right)
$$

and $J_{n}$ behaves like $x^{n}$ when $x>0$ is small. If $n$ is a negative integer, by definition yields

$$
J_{-n}(x)=(-1)^{n} J_{n}(x) .
$$

If $n$ is not an integer then

$$
J_{n}(x)=\frac{x^{n}}{2^{n} \Gamma(n+1)}\left(1-\frac{x^{2}}{2 \cdot(2 n+2)}+\frac{x^{4}}{2 \cdot 4 \cdot(2 n+2) \cdot(2 n+4)}-\ldots\right),
$$

where $\Gamma$ denotes the Gamma-function. When $n$ is an integer it is necessary to recall the so-called Weber's function, i.e.

$$
Y_{n}(x)=J_{n}(x) \int \frac{d x}{x J_{n}^{2}(x)}
$$

which behaves like $1 / x^{n}$ when $x>0$ is small. Next, we consider the Bessel equation

$$
y_{t t}-(2 \alpha-1) \frac{y_{t}}{t}+\left(\beta^{2} \tau^{2} t^{2 \tau-2}+\frac{\alpha^{2}-n^{2} \tau^{2}}{t^{2}}\right) y=0,
$$

Due to [7, pp. 117, the general solution of (3.36) is given by

$$
\begin{gathered}
y=t^{\alpha}\left\{A J_{n}\left(\beta t^{\tau}\right)+B Y_{n}\left(\beta t^{\tau}\right)\right\}, \\
y=t^{\alpha}\left\{A J_{n}\left(\beta t^{\tau}\right)+B J_{-n}\left(\beta t^{\tau}\right)\right\},
\end{gathered}
$$

where $A, B$ are constants, according as $n$ is non-negative integer or not.

Once $\lambda \neq 0$ is an eigenvalue for (3.33) then $\lambda$ is also an eigenvalue in (3.34). The general solution of (3.34) is a particular case of (3.36) for $\alpha=(N-1) / 2, \tau=1, \beta=\sqrt{\lambda}, n=$ $(N-3) / 2$, i.e.,

$$
v(t)=t^{\frac{N-1}{2}}\left\{A J_{\frac{N-3}{2}}(\sqrt{\lambda} t)+B Y_{\frac{N-3}{2}}(\sqrt{\lambda} t)\right\},
$$


or

$$
v(t)=t^{\frac{N-1}{2}}\left\{A J_{\frac{N-3}{2}}(\sqrt{\lambda} t)+B J_{-\frac{N-3}{2}}(\sqrt{\lambda} t)\right\} .
$$

We show that it must be $B=0$, in which case it simplifies the expression of $v$ i.e.

$$
v(t)=A t^{\frac{N-1}{2}} J_{\frac{N-3}{2}}(\sqrt{\lambda} t) .
$$

Indeed, if $N=3$ then, reconsidering the constants, it is trivial that $v$ is as in (3.37). Assume $N \geq 4$ and $B \neq 0$. Using the behavior of $J_{n}$ and $Y_{n}$ at zero we get that

$$
v(t) \sim A C_{1} t^{N-1}+B C_{2} t,
$$

where $C_{1}=C_{1}(\lambda), C_{2}=C_{2}(\lambda)$ are non-trivial constants depending on $\lambda$. Consequently, up to a constant,

$$
u(t) \sim 1+C t^{3-N}
$$

with $C \neq 0$. Since $N \geq 4$, this last formula yields to

$$
\int_{0}^{\gamma} u^{2}(t) d t=\infty
$$

which contradicts the fact that $u \in H$. Hence, the assumption is false and $B=0$ for any $N \geq 4$.

Imposing the condition $v(\gamma)=0$ in the simplified expresion (3.37), we obtain $\sqrt{\lambda}=B_{n}$, where $\left\{B_{n}\right\}_{n}$ are the positive zero's of the Bessel function $J_{\frac{N-3}{2}}$. In particular we obtain

$$
\lambda_{\star}^{1}(\gamma)=B_{1}^{2} / \gamma^{2} .
$$

Using this, the relations (3.11), (3.28) and the inequality (3.27) we obtain the conclusion of Theorem 3.2 .

\subsection{Proofs of useful results.}

Proposition 3.2. For any $v \in H$ we have

$$
\int_{0}^{\gamma} v_{t}^{2} t^{N-2} d t \geq \frac{1}{\gamma}\left(\frac{N-2}{2}\right)^{2} \int_{0}^{\gamma} v^{2} t^{N-3} d t
$$

Proof of Proposition (3.2). Of course, we have

$$
\int_{0}^{\gamma} v_{t}^{2} t^{N-2} d t \geq \frac{1}{\gamma} \int_{0}^{\gamma} v_{t}^{2} t^{N-1} d t
$$

and applying the Hardy inequality in $N$-d we complete the proof of Proposition (3.2).

Proposition 3.3. The embedding

$$
H \hookrightarrow L^{2}\left([0, \gamma], t^{N-2} d t\right)
$$

is compact.

Proof of Proposition 3.3. The key point is played by Proposition 3.2.

Next we consider a sequence $\left(u_{n}\right)_{n} \rightarrow 0$ in $H$ and suffices to prove its strong convergence in $L^{2}\left([0, \gamma], t^{N-2} d t\right)$ i.e. $u_{n} \rightarrow 0$ in $L^{2}\left([0, \gamma], t^{N-2} d t\right)$. By weak convergence, $\left\{u_{n}\right\}_{n}$ is bounded 
in $H_{0}^{1}\left([0, \gamma], t^{N-2} d t\right)$, let's say by a constant $C$. Accordingly to Proposition 3.2 we have as well

$$
\int_{0}^{\gamma} u_{n}^{2} t^{N-3} d t \leq C, \quad \forall n \in \mathbb{N} .
$$

Given $\varepsilon>0$ we split the $L^{2}$-norm by distinguish that concentrated in $B(0, \varepsilon)$ and in its exterior:

$$
\left\|u_{n}\right\|_{L^{2}\left([0, \gamma], t^{N-2} d t\right)}^{2}=\int_{0}^{2 \varepsilon}\left|u_{n}\right|^{2} t^{N-2} d t+\int_{2 \varepsilon}^{\gamma}\left|u_{n}\right|^{2} t^{N-2} d t:=I_{\varepsilon, n}^{1}+I_{\varepsilon, n}^{2} .
$$

Let us also consider the partition of unity of $u_{n}$,

$$
u_{n}=u_{n} \varphi+(1-\varphi) u_{n}:=w_{1, n}+w_{2, n},
$$

where $\varphi$ is a regular function such that

$$
\varphi(t)=\left\{\begin{array}{l}
1, \quad t \leq \varepsilon \\
0, \quad t \geq 2 \varepsilon
\end{array}\right.
$$

Obviously, $\operatorname{supp}\left(w_{1, n}\right) \subset(0,2 \varepsilon), \operatorname{supp}\left(w_{2, n}\right) \subset(\varepsilon, \gamma)$. Firstly, from (3.39) we have

$$
I_{\varepsilon, n}^{1} \leq 2 \varepsilon \int_{0}^{2 \varepsilon}\left|u_{n}\right|^{2} t^{N-3} d t \leq 2 \varepsilon \int_{0}^{\gamma}\left|u_{n}\right|^{2} t^{N-3} d t
$$

Secondly, let us notice that

$$
w_{2, n} \rightarrow 0 \text { in } H_{0}^{1}\left([0, \gamma], t^{N-2} d t\right) .
$$

For this, it suffices to prove $\left(\psi, w_{2, n}\right)_{H_{0}^{1}\left([0, \gamma], t^{N-2} d t\right)} \rightarrow 0$ for all $\psi \in C_{c}^{\infty}$. We evaluate,

$$
\left(\psi, w_{2, n}\right)_{H_{0}^{1}\left([0, \gamma], t^{N-2} d t\right)}=\int_{0}^{\gamma} \psi_{t}\left((1-\varphi) u_{n}\right)_{t} t^{N-2} d t=-\int_{0}^{\gamma}\left(\psi_{t} t^{N-2}\right)_{t}(1-\varphi) u_{n} d t
$$

which converges to 0 when $n \rightarrow \infty$. This happens because weak convergence in $H$ involves weak convergence in $L^{2}\left([0, \gamma], t^{N-3} d t\right)$ (by Proposition (3.2) ). Now we observe that the support of $w_{2, n}$ lies far from zero and therefore the norm of $w_{2, n}$ in $H$ is equivalent to the norm of $w_{2, n}$ in $H_{0}^{1}(\varepsilon, \gamma)$. But $H_{0}^{1}(\varepsilon, \gamma)$ is compact embedded in $L^{2}(\varepsilon, \gamma)$, and in particular in $L^{2}\left([0, \gamma], t^{N-3} d t\right)$. We obtain that $w_{2, n} \rightarrow 0$ in $L^{2}\left([0, \gamma], t^{N-3} d t\right)$. Hence, we can choose $n$ large enough such that $I_{\varepsilon, n}^{2}<\varepsilon$. From here and (3.42), we conclude that $u_{n}$ converges strongly to 0 in $L^{2}\left([0, \gamma], t^{N-2} d t\right)$.

Lemma 3.1. Let us consider $a<1$. Then, the application

$$
\left(0, \frac{\pi}{2}\right) \text { э } t \rightarrow \frac{\tan a t}{\tan t} \in(0, \infty)
$$

is decreasing.

Proof of Lemma 3.1. Indeed, if we consider $f(t)=\frac{\tan a t}{\tan t}$ we obtain

$$
f^{\prime}(t)=\frac{a t}{\cos ^{2} a t \sin ^{2} t}\left(\frac{\sin 2 t}{2 t}-\frac{\sin 2 a t}{2 a t}\right) .
$$

It follows that $f^{\prime}<0$ due to the decreasing behavior of the function $x \mapsto \frac{\sin x}{x}$ on $(0, \pi)$.

With this, we complete the proof. 
Lemma 3.2. For any $\varepsilon>0, \varepsilon<<1$, there exists $\delta=\delta(\varepsilon)>0$ such that

$$
\sin \left(\frac{\pi}{2 \gamma(\varepsilon)} \theta_{1}\right) \cos \theta_{1} \geq(1-\varepsilon) \cos \left(\frac{\pi}{2 \gamma(\varepsilon)} \theta_{1}\right) \sin \theta_{1}, \quad \forall \quad 0 \leq \theta_{1} \leq \frac{\pi}{2}-\varepsilon .
$$

Proof of Lemma 3.2. Let us put formally $\gamma=\pi / 2+\delta$ with $\delta>0$. Then (3.44) becomes

or

$$
\sin \left(\frac{\pi}{\pi+2 \delta(\varepsilon)} \theta_{1}\right) \cos \theta_{1} \geq(1-\varepsilon) \cos \left(\frac{\pi}{\pi+2 \delta(\varepsilon)} \theta_{1}\right) \sin \theta_{1}
$$

or equivalent to

$$
\tan \left(\frac{\pi}{\pi+2 \delta(\varepsilon)} \theta_{1}\right) \geq(1-\varepsilon) \tan \theta_{1}, \quad \forall \quad 0 \leq \theta_{1} \leq \pi / 2-\varepsilon,
$$

$$
\frac{\tan \left(\frac{\pi}{\pi+2 \delta(\varepsilon)} \theta_{1}\right)}{\tan \theta_{1}} \geq(1-\varepsilon), \quad \forall \quad 0 \leq \theta_{1} \leq \pi / 2-\varepsilon .
$$

Now, let us show the validity of (3.45) . Because

$$
\lim _{\delta \searrow 0} \frac{\tan \left(\frac{\pi}{\pi+2 \delta}\left(\frac{\pi}{2}-\varepsilon\right)\right)}{\tan \left(\frac{\pi}{2}-\varepsilon\right)}=1,
$$

we can choose $\delta=\delta(\varepsilon)>0$ such that

$$
\frac{\tan \left(\frac{\pi}{\pi+2 \delta(\varepsilon)}\left(\frac{\pi}{2}-\varepsilon\right)\right)}{\tan \left(\frac{\pi}{2}-\varepsilon\right)} \geq(1-\varepsilon) .
$$

By this inequality and Lemma 3.1 it is easy to obtain (3.45).

Lemma 3.3. Let $0<\varepsilon<<1$. There exists $\delta=\delta(\varepsilon)>0$ and a constant $C$, such that

$$
\left|\frac{\cos \theta_{1}}{\cos \left(\frac{\pi}{2 \gamma(\varepsilon)} \theta_{1}\right)}\right|<C, \quad \forall \quad 0 \leq \theta_{1} \leq \bar{\gamma}(\varepsilon),
$$

Proof of Lemma 3.3. Let us put $\theta_{1}=\pi / 2+\delta(\varepsilon) t^{\prime \prime}$ with $t^{\prime \prime} \in(0,1 / 2)$. Then

$$
\left|\frac{\cos \theta_{1}}{\cos \left(\frac{\pi}{2 \gamma(\varepsilon)} \theta_{1}\right)}\right|=\left|\frac{\sin \delta(\varepsilon) t^{\prime \prime}}{\sin \left(\frac{\pi \delta(\varepsilon)}{\pi+2 \delta(\varepsilon)}\left(1-t^{\prime \prime}\right)\right)}\right| \rightarrow \frac{t^{\prime \prime}}{1-t^{\prime \prime}},
$$

when $\delta(\varepsilon) \rightarrow 0, \quad \forall \quad t^{\prime \prime} \in(0,1 / 2)$. But

$$
\sup _{t^{\prime \prime} \in(0,1 / 2)} \frac{t^{\prime \prime}}{1-t^{\prime \prime}}=1
$$

and this yields to the conclusion of Lemma.

\section{Further comments And OPEn PRoblems}

4.1. Efficiency of the methods and sharp reminder terms. As we mentioned in Theorem [2.1, the inequality we obtained involves a reminder term of order $\int v^{2} /|x| d x$ in the lower bound. The proof uses a change of variable adapted to the boundary near the singular point. Comparing Theorems 2.1 and 2.2, we see that the results improve when using spherical harmonics decomposition. More precisely, the inequality stated in Theorem 2.2 admits an optimal reminder term of order $\int v^{2} /\left(|x|^{2} \log ^{2}(1 /|x|)\right) d x$. Thus, spherical harmonics decomposition yields better results. 
4.2. Inequalities in cones. In 2 - $\mathrm{d}$ we have given a complete picture of the sharp Hardy inequality. In the multi-dimensional case, $N \geq 3$, we proved several qualitative inequalities but optimality results are still to be proved. To be more precise, in convex cones we proved that the constant is at least $(N-2)^{2} / 4+(N-1) \pi^{2} / 4 \gamma^{2}, \gamma$ being the slot of the cone (see Subsection 3.1). This result shows that the best constant blows-up when $\gamma$ tends to 0. For concave cones we have obtained less information: we have only shown that the constant varies continuously with respect to the slot $\gamma$ nearby $\gamma=\pi / 2$. For any cone with the slot $\gamma \in(0, \pi)$ we have proved that the best constant is at least $(\sin \gamma / \gamma)^{N-2} B_{1}^{2} / \gamma^{2}$, where $x_{1}$ is the first positive zero of the Bessel function $J_{(N-3) / 2}$. To our knowledge, explicit formulas for the optimal constant are still to be proved.

4.3. Weak Hardy inequalities with $L^{2}$-reminder terms. In the context of smooth domains, for certain geometries, we have improved the Hardy constant from $(N-2)^{2} / 4$ to $N^{4} / 4$. To do this, we had to add a $L^{2}$-reminder term in the upper bound of the inequality. This extra term in $L^{2}$-norm cannot be disregarded as shown in Proposition 2.1. Thus, the inequalities that we obtain are sharp. However, the problem on the possible existence of domains $\Omega$ satisfying $\mathrm{C} 3$ such that $\mu(\Omega)=N^{2} / 4$ is open. In the case where $\Omega$ has an hyperbolic geometry at the origin, as $\varepsilon$ tends to 0 , the constant $C(\Omega, \varepsilon)$ is expected to blow-up (see Theorem 2.4). This class of generalized Hardy inequalities with lower order reminder terms is of application in various contexts. For instance, the Hardy inequalities play a crucial role when studying the controllability of wave equations with quadratic singular potentials. In that setting, one can get rid of the $L^{2}$-reminder terms, using compactness-uniqueness arguments (see [14]).

Acknowledgements. I am really grateful to Enrique Zuazua for his guidance. I wish to thank Adi Adimurthi, David Krejcirik, Kyril Tintarev, Liviu Ignat, Alessio Porretta, for fruitful discussions.

\section{REFERENCES}

[1] N. C. Adimurthi and M. Ramaswamy, An improved Hardy-Sobolev inequality and its application, Proc. Amer. Math. Soc. 130 (2002), no. 2, 489-505 (electronic).

[2] N.C. Adimurthi and K. Sandeep, Existence and non-existence of the first eigenvalue of the perturbed Hardy-Sobolev operator, Proc. Roy. Soc. Edinburgh Sect. A 132 (2002), no. 5.

[3] G. Barbatis, S. Filippas, and A. Tertikas, A unifed approach to improved Lp Hardy inequalities with best constants, Trans. Amer. Math. Soc. 356 (2004), no. 6, 2169-2196 (electronic).

[4] H. Berestycki and M. J. Esteban, Existence and bifurcation of solutions for an elliptic degenerate problem, J. Differential Equations 134 (1997), no. 1, 1-25.

[5] W. M. Boothby, An introduction to differentiable manifolds and Riemannian geometry, second ed., Pure and Applied Mathematics, vol. 120, Academic Press Inc., Orlando, FL, 1986.

[6] R. Bosi, J. Dolbeault, and J. Esteban, M., Estimates for the optimal constants in multipolar Hardy inequalities for Schrödinger and Dirac operators, Commun. Pure Appl. Anal. 7 (2008), no. 3, 533-562.

[7] F. Bowman, Introduction to Bessel functions, Dover Publications Inc., New York, 1958.

[8] H. Brezis and M. Marcus, Hardy's inequalities revisited, Ann. Scuola Norm. Sup. Pisa Cl. Sci. (4) 25 (1997), no. 1-2, 217-237 (1998), Dedicated to Ennio De Giorgi.

[9] 9. H. Brezis, M. Marcus, and I. Shafrir, Extremal functions for Hardy's inequality with weight, J. Funct. Anal. 171 (2000), no. 1, 177-191.

[10] H. Brezis and J. L. Vázquez, Blow-up solutions of some nonlinear elliptic problems, Rev. Mat. Univ. Complut. Madrid 10 (1997), no. 2, 443-469.

[11] L. Caffarelli, R. Kohn, and L. Nirenberg, First order interpolation inequalities with weights, Compositio Math. 53 (1984), no. 3, 259-275. 
[12] P. Caldiroli and R. Musina, On a class of two-dimensional singular elliptic problems, Proc. Roy. Soc. Edinburgh Sect. A 131 (2001), no. 3, 479-497.

[13] P. Caldiroli and R. Musina, Stationary states for a two-dimensional singular Schrödinger equation, Boll. Unione Mat. Ital. Sez. B Artic. Ric. Mat. (8) 4 (2001), no. 3, 609-633.

[14] C. Cazacu and E Zuazua, Controllability of the wave equation with boundary singular quadratic potential, in preparation.

[15] C. Cazacu and E Zuazua, Hardy inequalities with boundary singular quadratic potential, Proceedings Picof10, Cartagena, Spain, 2010, 149-155.

[16] H. L. Cycon, R. G. Froese, W. Kirsch, and B. Simon, Schrödinger operators with application to quantum mechanics and global geometry, Berlin (1987).

[17] V. Felli and S. Terracini, Elliptic equations with multi-singular inverse-square potentials and critical nonlinearity , Comm. Partial Differential Equations 31 (2006), no. 1-3, 469-495.

[18] S. Filippas, V. Maz'ya, and A. Tertikas, On a question of Brezis and Marcus, Calc. Var. Partial Differential Equations 25 (2006), no. 4, 491-501.

[19] S. Filippas, V. G. Maz'ya, and A. Tertikas, Sharp Hardy-Sobolev inequalities, C. R. Math. Acad. Sci. Paris 339 (2004), no. 7, 483-486.

[20] Tertikas A. Filippas, S. and J. Tidblom, On the structure of Hardy-Sobolev-Maz'ya inequalities, J. Eur. Math. Soc. (JEMS) 11 (2009), no. 6, 1165-1185.

[21] S. Gallot, D. Hulin, and J. Lafontaine, Riemannian geometry, Universitext, Springer-Verlag, Berlin, 1987.

[22] J. P. García Azorero and A. I. Peral, Hardy inequalities and some critical elliptic and parabolic problems, J. Differential Equations 144 (1998), no. 2, 441-476.

[23] N. Ghoussoub and C. Yuan, Multiple solutions for quasi-linear PDEs involving the critical Sobolev and Hardy exponents, Trans. Amer. Math. Soc. 352 (2000), no. 12, 5703-5743.

[24] G. H. Hardy, J. E. Littlewood, and G. Pólya, Inequalities, Cambridge Mathematical Library, Cambridge University Press, Cambridge, 1988, Reprint of the 1952 edition.

[25] G.H. Hardy, An inequality between integrals, Messenger of Math. 54 (1925), 150-156.

[26] D. Krejcirik and E. Zuazua, The Hardy inequality and the heat equation in twisted tubes, J. Math. Pures Appl. (2010).

[27] J.M. Lévy-Leblond, Electron capture by polar molecules, Phys. Rev. 153 (1967), no. 1, 1-4.

[28] M. F. Mouhamed and R. Musina, Hardy-Poincaré inequalities with boundary singularities, http://arxiv.org/abs/1005.3400

[29] Y. Pinchover and K. Tintarev, Existence of minimizers for Schrödinger operators under domain perturbations with application to Hardy's inequality, Indiana Univ. Math. J. 54 (2005), no. 4, 1061-1074.

[30] E. M. Stein and R. Shakarchi, Fourier analysis, Princeton Lectures in Analysis, vol. 1, Princeton University Press, 2003.

[31] A. Tertikas, Critical phenomena in linear elliptic problems, J. Funct. Anal. 154 (1998), no. 1, 42-66.

[32] K. Tintarev and K. H. Fieseler, Concentration compactness, Imperial College Press, London, 2007.

[33] J. L. Vázquez and E. Zuazua, The Hardy inequality and the asymptotic behaviour of the heat equation with an inverse-square potential, J. Funct. Anal. 173 (2000), no. 1, 103-153. 